\title{
TEXS: in-vacuum tender X-ray emission spectrometer with 11 Johansson crystal analyzers - SUPPORTING INFORMATION
}

\author{
Mauro Rovezzi, ${ }^{1,2, \text { a) }}$ Alistair Harris, ${ }^{3}$ Blanka Detlefs, ${ }^{2}$ Timothy Bohdan, ${ }^{2}$ Artem Svyazhin, ${ }^{2,4}$ Alessandro \\ Santambrogio, ${ }^{2}$ David Degler, ${ }^{2}$ Rafal Baran, ${ }^{2}$ Benjamin Reynier, ${ }^{2}$ Pedro Noguera Crespo, ${ }^{5}$ Catherine \\ Heyman, ${ }^{3}$ Hans-Peter Van Der Kleij, ${ }^{2}$ Pierre Van Vaerenbergh, ${ }^{2}$ Philippe Marion, ${ }^{2}$ Hugo Vitoux, ${ }^{2}$ Christophe \\ Lapras, ${ }^{2}$ Roberto Verbeni, ${ }^{2}$ Menhard Menyhert Kocsis, ${ }^{2}$ Alain Manceau, ${ }^{6}$ and Pieter Glatzel ${ }^{2, b)}$ \\ ${ }^{1)}$ Univ. Grenoble Alpes, CNRS, IRD, Irstea, Météo France, OSUG, FAME, 71 avenue des Martyrs, CS 40220, 38043, Grenoble, \\ France \\ ${ }^{2)}$ European Synchrotron Radiation Facility, 71 avenue des Martyrs, CS 40220, 38043, Grenoble, \\ France \\ ${ }^{3)}$ Design et Mécanique, Les Coings, 38210, Montaud, France \\ ${ }^{4)}$ M. N. Miheev Institute of Metal Physics, Ural Branch of the Russian Academy of Science, 620990, Ekaterinburg, \\ Russia \\ ${ }^{5)}$ Added Value Solutions (AVS), Pol. Ind. Sigma Xixilion Kalea 2, Bajo Pabellón 10, 20870, Elgoibar, \\ Spain \\ ${ }^{6}$ ISTerre, Université Grenoble Alpes, CNRS, CS 40700, 38058, Grenoble, France \\ (Dated: April 8, 2020)
}

\section{S1. DETECTOR ALTERNATIVES TO THE PROPORTIONAL COUNTER}

We examined other solutions for the detector among existing commercial alternatives or promising projects in development. The most suited detector for such an application would be a scientific-quality large area charged-coupled device (CCD). CCDs are widely used in soft X-ray RIXS spectrometers for their low electronic noise and small pixel size. Furthermore, it is possible to run them in spectroscopy mode ${ }^{\mathrm{S} 1}$, enhancing the possibility to reduce the background level of the measured signal. Commercial sensors have a standard size of approximately $27 \times 27 \mathrm{~mm}^{2}$, that is, 2048 square pixels of $13 \mu \mathrm{m}$ side. The drawback of such sensors is the read-out time, being as slow as $500 \mathrm{~ms}$ for unbind mode, well above the required $2 \mathrm{~ms}$. Fast readout and large area CCDs exist, like the pnCCD ${ }^{\mathrm{S} 2}$ or the fastCCD ${ }^{\mathrm{S} 3}$. These extremely high performance detectors, initially developed for astronomical applications in space telescopes, were out of our budget. An alternative to CCDs are silicon drift detectors (SDDs). Typical sensors sizes are of $50 \mathrm{~mm}^{2}$ area, thus to cover the required area of $2000 \mathrm{~mm}^{2}$ one could opt for a multi-element array $^{\mathrm{S} 4}$ or pixelated sensors ${ }^{\mathrm{S} 5}$. Nevertheless, this solution is challenging and was not available at the time of the design. Hybrid pixel detectors, that is, a pixelated sensor (usually $\mathrm{Si}$ ) bump-bonded to a large area CMOS have also demonstrated some applications in the low energy side ${ }^{\mathrm{S} 6, \mathrm{~S} 7}$. Recently, direct reading of mass production CMOS cameras was shown as a possible alternative for tender $\mathrm{X}$-rays ${ }^{\mathrm{S} 8, \mathrm{~S} 9}$. The drawback of these CMOS-based detectors is their quantum efficiency, usually below $20 \%$. In fact, either the high noise level imposes an electronic threshold that barely allows the signal to be measured (the case of hybrid pixel detectors) or the active layer of the CMOS is too thin (the case of direct CMOS reading).

\footnotetext{
a) Electronic mail: mauro.rovezzi@esrf.fr

b)Electronic mail: glatzel@esrf.fr
}

\section{S2. NAMING CONVENTION FOR WIRE SIGNALS AND ANALYZER MODULES}

The naming convention for the detector wires $(\mathrm{gN})$ and analyzers modules (athN) is given in Figure S1. The figure allows understanding the origin of the signal detected on each wire and the arrangement of the analyzers with respect to the incoming $\mathrm{X}$-ray beam ( $\mathrm{X}$ direction).

\section{S3. DETECTOR TRAJECTORIES AND HOSES}

The trajectories for $2 \mathrm{R}=1020 \mathrm{~mm}$ and $2 \mathrm{R}=480 \mathrm{~mm}$, respectively, maximum and minimum allowed diameter of the Rowland circle are shown in Figure S2. The simulation of the hoses position at given angular positions is also shown. Those simulations have been used for testing the behaviour of the metallic hoses and measuring the forces on the detector's arm, as shown in Figure S3.

\section{S4. GAS DETECTOR EFFICIENCY}

The gas detector efficiency, that is, the $\mathrm{X}$-ray absorption (in the energy range of interest) by the $15 \% \mathrm{CO}_{2}$ in Ar mixture at $1 \mathrm{bar}$ and at room temperature in the $4 \mathrm{~cm}$ path is shown in Figure S4.

\section{S5. GAS DETECTOR LINEARITY}

The gas detector linearity as a function of the incoming beam flux is measured for each wire at each experimental session, as shown in Figure S5. The intensity of the incoming beam is varied by closing the incoming beam slits or scanning the undulators' gap. In fact, in the tender X-ray energy range the use of filters to attenuate the beam is not possible because of their strong absorption. A proportional signal is 

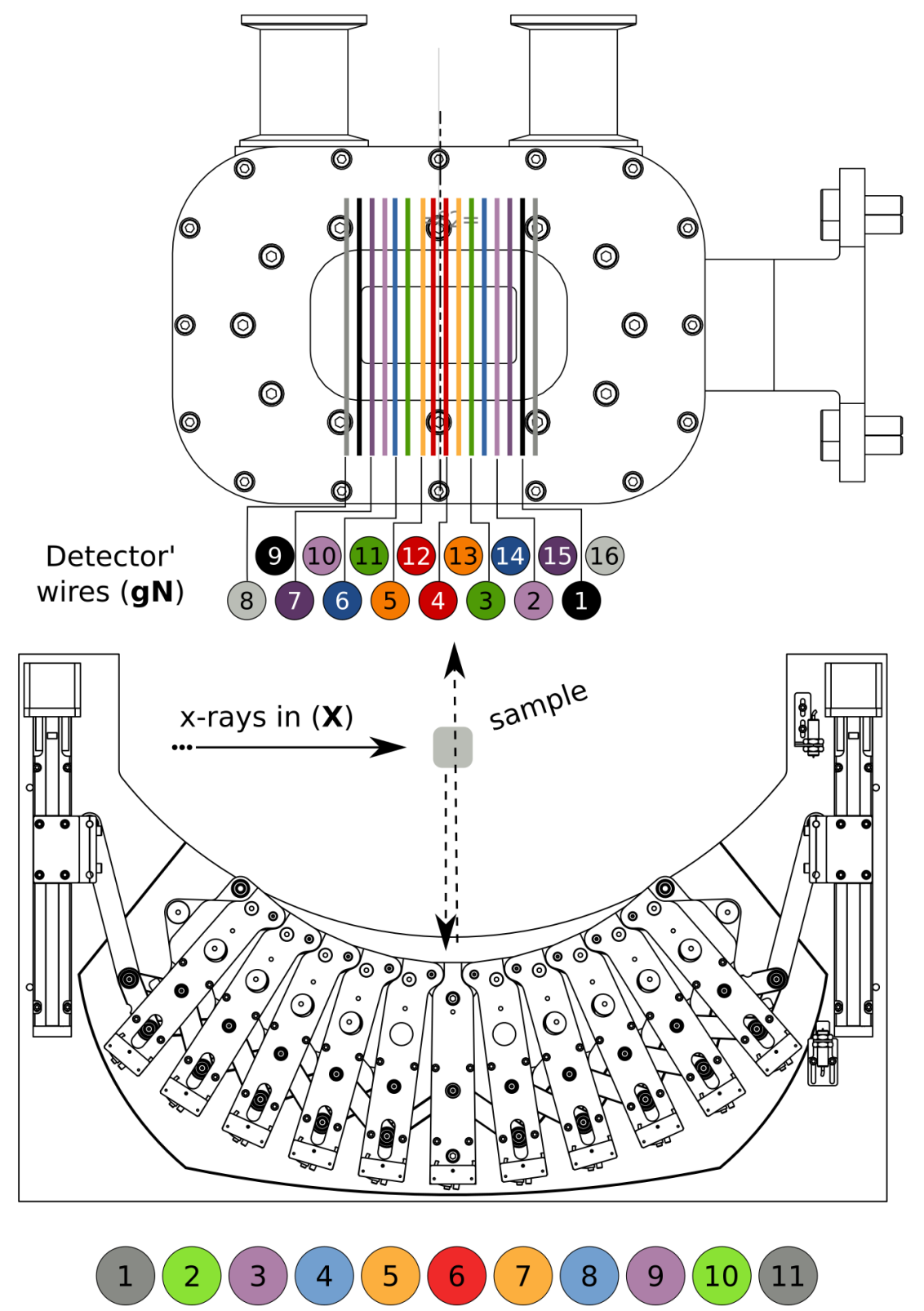

Analysers (athN)

Figure S1. Naming of the detector wires signals ( $\mathrm{gN}$ ) and analyzer modules (athN) used during the commissioning and data analysis. Detector and analyzer table are given in a projected view such as looking from the analyzer door of the spectrometer, that is, the incoming X-ray beam is coming from the left side. Dimensions are not to scale. An independent colour coding for the analyzer table and detector is used to show the central symmetry.

measured via the current of a Silicon photo-diode reading the back-scattering signal from a Kapton foil of $8 \mu \mathrm{m}$ thickness (Figure S6C.4). The dead time constant $(\tau)$ for each wire of the gas detector is found by fitting the response in a linear region, that is, below $25 \mathrm{kHz}$ (counts/s) per wire, using the standard formula of the nonparalyzable model (S10, pag. 122): $m=k n /(1+k n \tau)$, where $m$ is the gas detector signal in the linear region, $n$ is the photo-diode signal, assumed as the true signal of the incoming beam, and $k$ a constant. In fact, the absolute number of counts of the photo-diode are not the absolute number of incoming photons/s. 


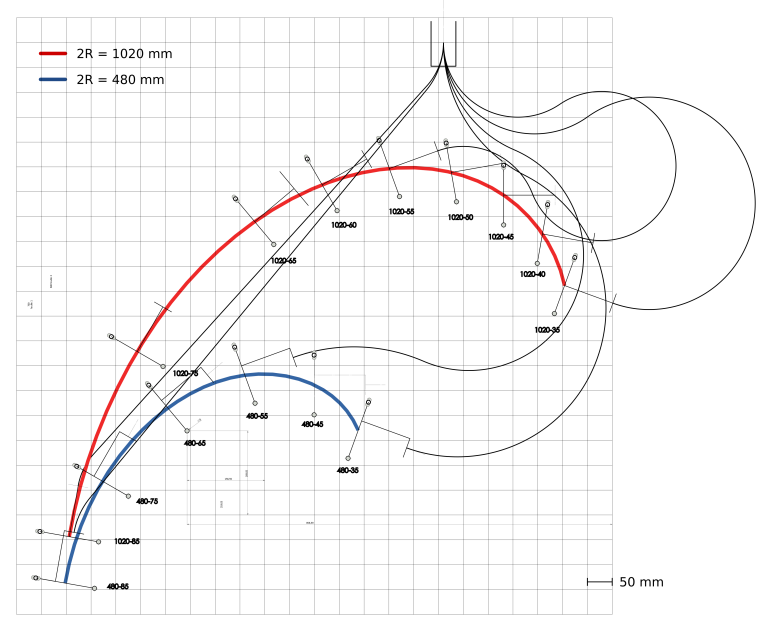

Figure S2. Trajectories of the detector chamber with the simulation of the hoses movements for two extreme radii.

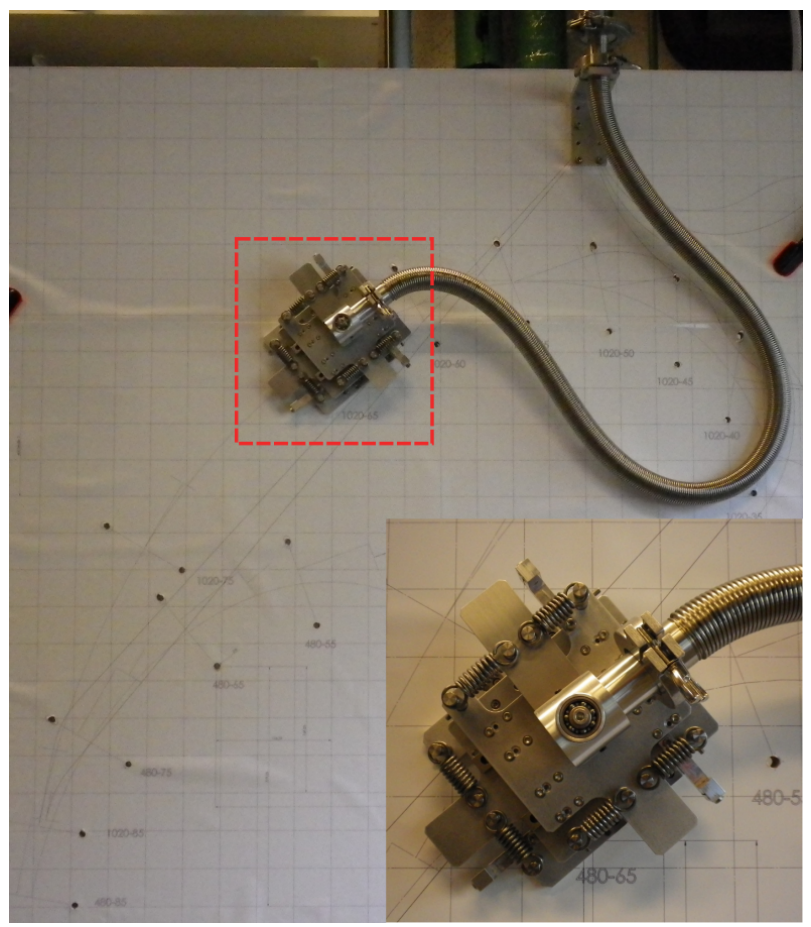

Figure S3. Prototype mechanics for testing the metallic hoses in real conditions and measuring the forces acting on the detector arm.

\section{S6. SAMPLE ENVIRONMENT}

The sample environment space is shown in Figure S6. Examples of the sample holders are shown in Figure S7.

\section{S7. OPERANDO CELL FOR GAS SENSORS}

In-situ and operando XAS are an essential method to understand synthesis-structure-function-relationships of metal ox-

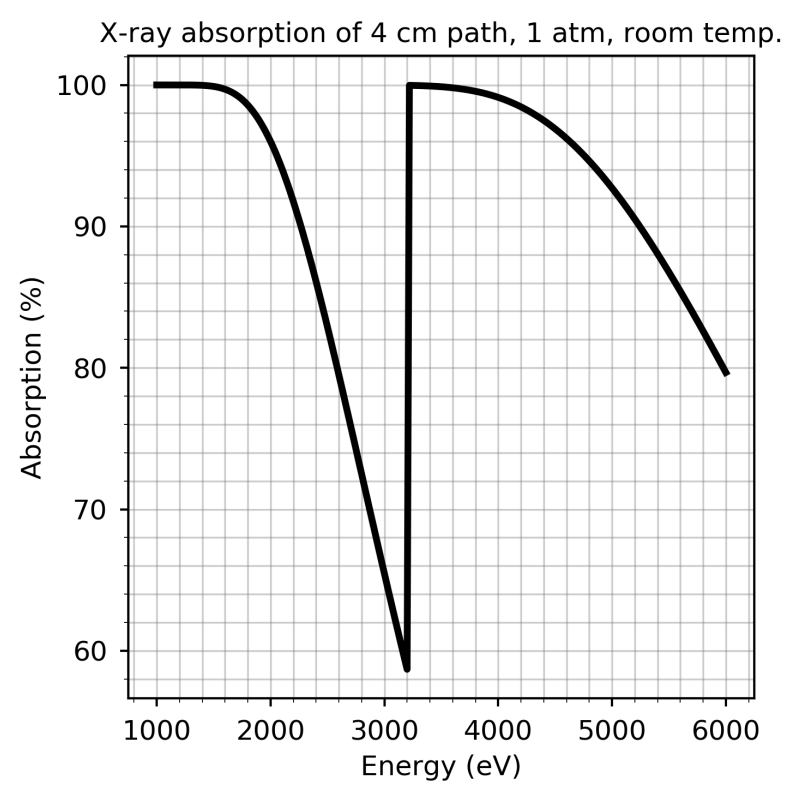

Figure S4. Gas detector efficiency in the energy range of interest.

ide based gas sensing materials ${ }^{\mathrm{S} 11, \mathrm{~S} 12}$. The elements of interest are those from base materials such as $\mathrm{SnO}_{2}, \mathrm{In}_{2} \mathrm{O}_{3}$ and $\mathrm{WO}_{3}$, and additives like $\mathrm{Rh}, \mathrm{Pd}, \mathrm{Pt}$ and $\mathrm{Au}$. In case of $5 d$-elements there are various examples using HERFD-XAS to study the behavior of additives during gas sensing ${ }^{\mathrm{S} 13, \mathrm{~S} 14}$, while in case of $4 d$-elements $\mathrm{K}$-edges were studied using conventional XAS ${ }^{\mathrm{S} 15-\mathrm{S} 17}$. Extending the spectral range to tender $\mathrm{X}$-rays allows studying the L-edges of $4 d$-elements and will provide unprecedented insights in the gas sensing process of pristine, doped and loaded metal oxide gas sensing materials.

For TEXS a suitable operando gas sensor cell (Figure S8A) was designed, which allows dynamically dosing different gas flows. The sensor socket is designed to mount research-type gas sensor substrates ${ }^{\mathrm{S} 18}$ and is equipped with electrical connections to supply a heating voltage and continuously read the sensor response during the experiment. Successful tests were done studying a $2 \mathrm{wt} \% \mathrm{Pd}$-loaded $\mathrm{SnO}_{2}$ gas sensor at $300{ }^{\circ} \mathrm{C}$ in different atmospheres (Figure S8B). The recorded HERFDXANES spectra show a clear effect of the atmospheric composition on the Pd sites.

\section{S8. INITIAL CHARACTERIZATION OF COMMERCIAL ANALYZERS}

A brief summary of the characterization of two selected commercial Johansson crystal analyzers (double machining) and one produced at the ESRF (single machining) is shown in Figure S9. The measurements were performed on the high energy spectrometer of ID26 at $8186 \mathrm{eV}$, using $\mathrm{Si}(444)$ reflection at a Bragg angle of $75^{\circ}$, following a well established procedure ${ }^{\mathrm{S} 19}$. In summary, the elastic scattering peaks are collected at the best bending radius by scanning the energy of the incoming beam with a cryogenically cooled dou- 

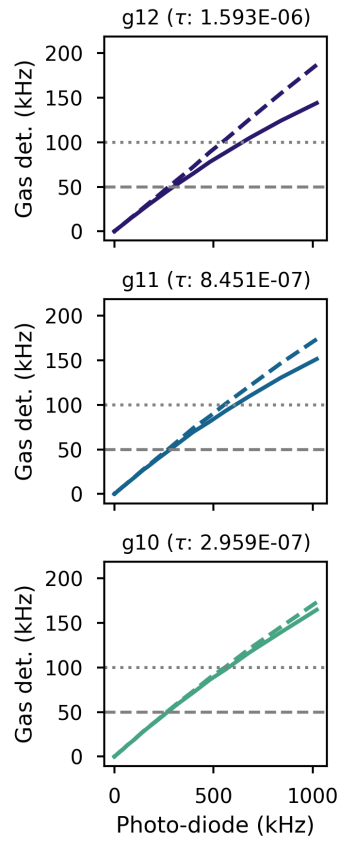
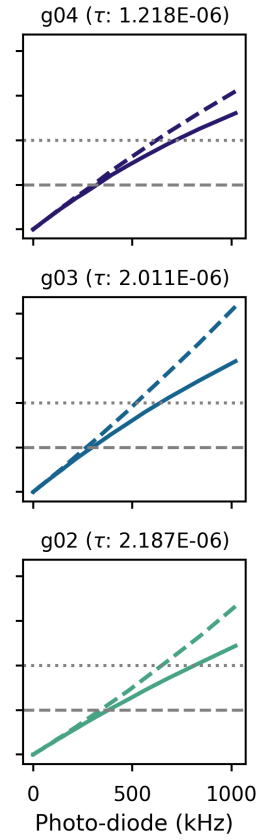
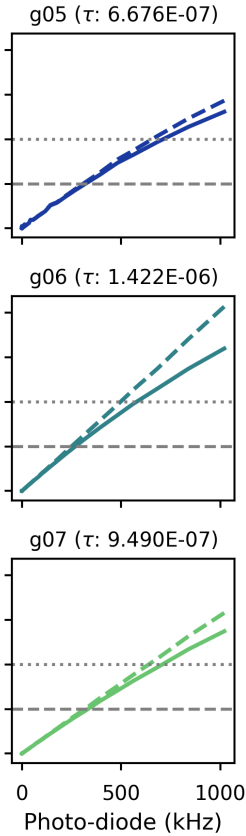
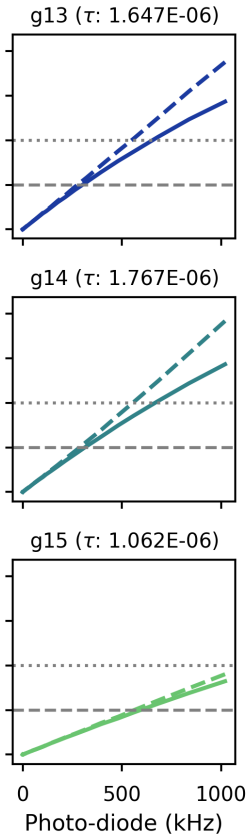

Figure S5. Typical single-wire signal response of the gas detector as a function of the incoming beam flux (photo-diode signal) in kHz (1000 counts/s). The dashed lines are the dead-time corrected signal, using a dead-time constant $(\tau)$ fitted in the linear response region below $25 \mathrm{kHz}$. The two horizontal dashed and dotted lines are a guide for the eye, respectively, at $50 \mathrm{kHz}$ and $100 \mathrm{kHz}$.

ble $\mathrm{Si}(311)$ monochromator. During these measurements, a square avalanche photo diode detector of $10 \mathrm{~mm}$ side was used. This means that only a central area of $5 \mathrm{~mm}$ side along the flat direction was probed. The energy bandwidth measured with the "COM 1" analyzer corresponds to what is expected by a ray tracing simulation, that is, the experimental curve overlaps the simulated (normalized to peak maximum). The "COM 2" analyzer shows higher reflectivity, with an integrated area almost four times the area of "COM 1". The ESRF analyzer corresponds to a first production series of singlemachined analyzers, that is, without grooves. This explains the lower performance with respect to the commercial analyzers. The grooves and the optimization of the surface polishing (as reported in the main text) have permitted to remove the side tails visible in the reflectivity curve.

\section{S9. MACHINING CYLINDRICAL JOHANSSON CRYSTAL ANALYZERS}

The two standard machining approaches in the production of cylindrical Johansson crystal analyzers, namely, single and double machining, are schematized in Figure S10.

\section{S10. MEASURED EMISSION LINES}

The measured emission lines reported in Table 1 of the main text are shown in Figure S11.

\section{S11. EXAMPLE OF THE DATA ANALYSIS WORKFLOW}

An example of the data analysis workflow, that is, the procedure we have employed to extract the instrumental energy resolution reported in Table 1 of the main text, is shown here for the $\mathrm{S} \mathrm{K} \alpha_{1,2}$ lines (Figure S12). It is implemented in Python as a series of Jupyter notebooks and available on demand. The naming convention is given in Figure S1. The first step of the analysis consists in a peak fitting (Figure S12A): for each crystal analyzer mounted on the 11 holders of the spectrometer, the signal of each wire out of the sixteen available of the gas detector is analyzed independently. This corresponds to an average statistics of approximately 100 fits, out of the 176 possible, as not all wires read a good signal for each scan. In the second step (Figure S12B), each property of the peak fitting, here the experimental full-width-at-halfmaximum of the main peak (p1_fwhm_exp) is plotted per analyzer and following the spatial distribution of the wires. This step allows us to establish any issue related to mis-alignment of the spectrometer. It also allows to reject outliers. If no major problems are found, the results are taken all together (Figure S12C) to establish the average reported value and the experimental standard deviation (error bar). We note that the error bar of the fit is negligible with respect to the experimental standard deviation. 


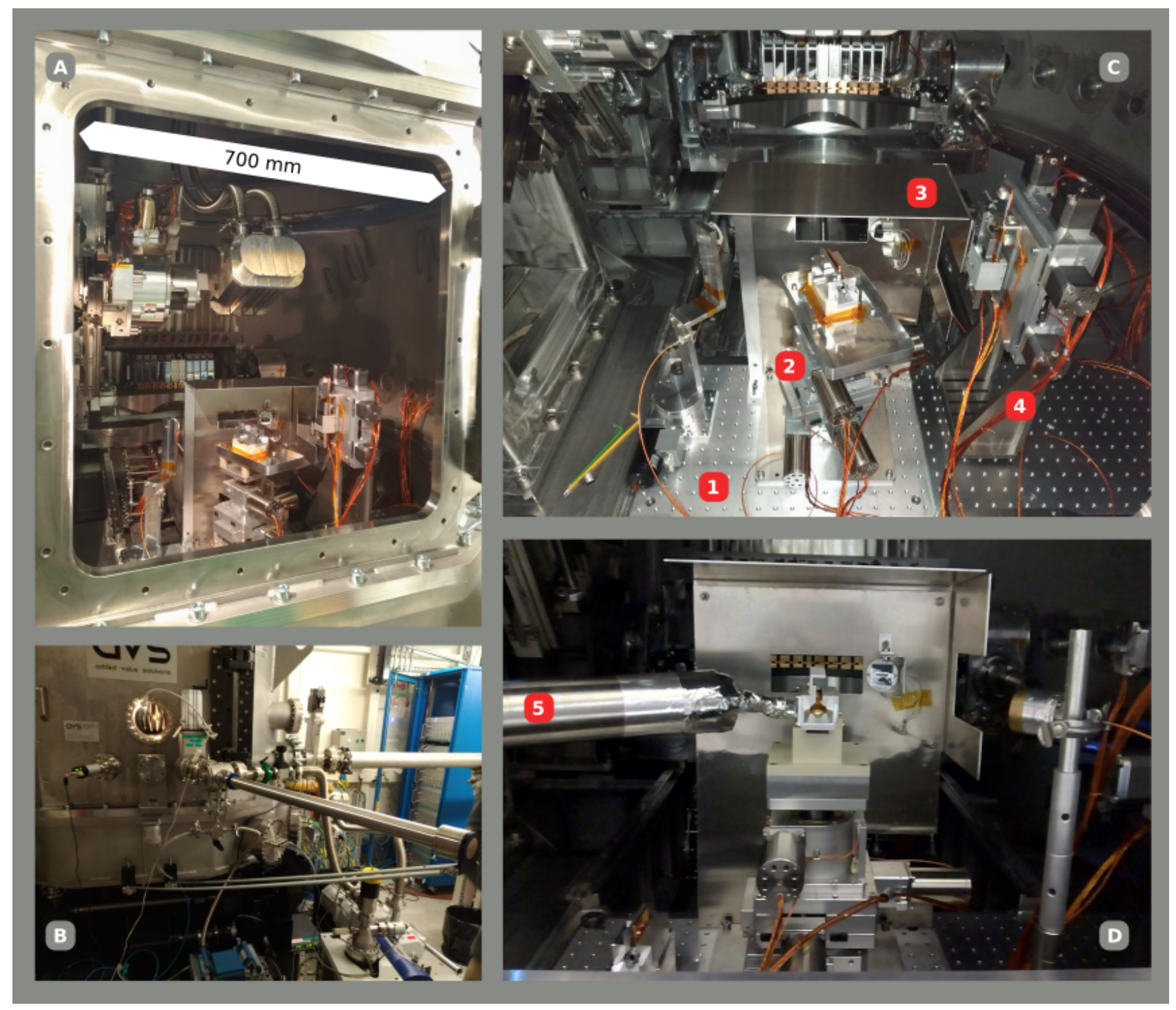

Figure S6. Sample environment space. Front door (A). External view of the load-lock system (B). Internal views (C and D): base breadboard (1), sample tower (2), shielding for blocking background signals (3), incoming beam slits and intensity monitor (4), head of the liquid helium cryostat (5).

\section{S12. BEST RADIUS OPTIMISATION PROCEDURE}

The procedure employed for finding the best Rowland circle radius parameter $(\mathrm{R})$ for each analyzer is summarized in Figure S13.

\section{REFERENCES}

${ }^{\mathrm{S} 1}$ W. Zhao and K. Sakurai, Review of Scientific Instruments 88, 063703 (2017).

${ }^{S}$ L. Strüder, Nuclear Instruments and Methods in Physics Research Section A: Accelerators, Spectrometers, Detectors and Associated Equipment 454, 73 (2000).

${ }^{S}$ D. Doering, Y.-D. Chuang, N. Andresen, K. Chow, D. Contarato, C. Cummings, E. Domning, J. Joseph, J. S. Pepper, B. Smith, G. Zizka, C. Ford, W. S. Lee, M. Weaver, L. Patthey, J. Weizeorick, Z. Hussain, and P. Denes, Review of Scientific Instruments 82, 073303 (2011).

${ }^{S 4}$ I. Hafizh, G. Bellotti, M. Carminati, G. Utica, M. Gugiatti, A. Balerna, V. Tullio, G. Lepore, G. Borghi, F. Ficorella, A. Picciotto, N. Zorzi, A. Capsoni, S. Coelli, L. Bombelli, and C. Fiorini, Journal of Instrumentation 14, P06027 (2019).

${ }^{5}{ }^{5}$ J. Bufon, M. Ahangarianabhari, P. Bellutti, G. Bertuccio, S. Carrato, G. Cautero, S. Fabiani, G. Giacomini, A. Gianoncelli, D. Giuressi, M. Grassi, P. Malcovati, R. Menk, A. Picciotto, C. Piemonte, I. Rashevskaya, A. Rachevski, A. Stolfa, A. Vacchi, G. Zampa, and N. Zampa, 

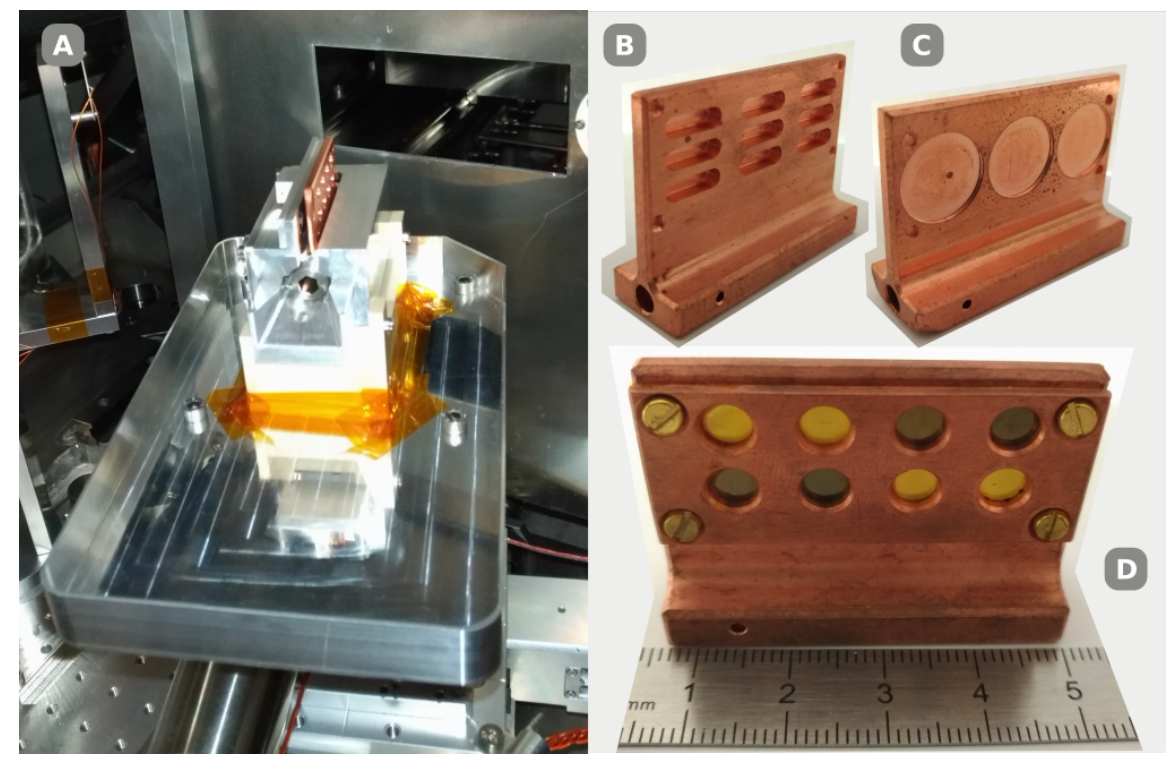

Figure S7. Receptacle of the sample holder at the top of the sample goniometer tower (A). Example of sample holders copper blocks: Cells for frozen liquids (B), circular pellets of $13 \mathrm{~mm}$ diameter (C) and $5 \mathrm{~mm}$ diameter, showing eight mounted pellets sealed with a Kapton foil kept in place by a front mask (D).
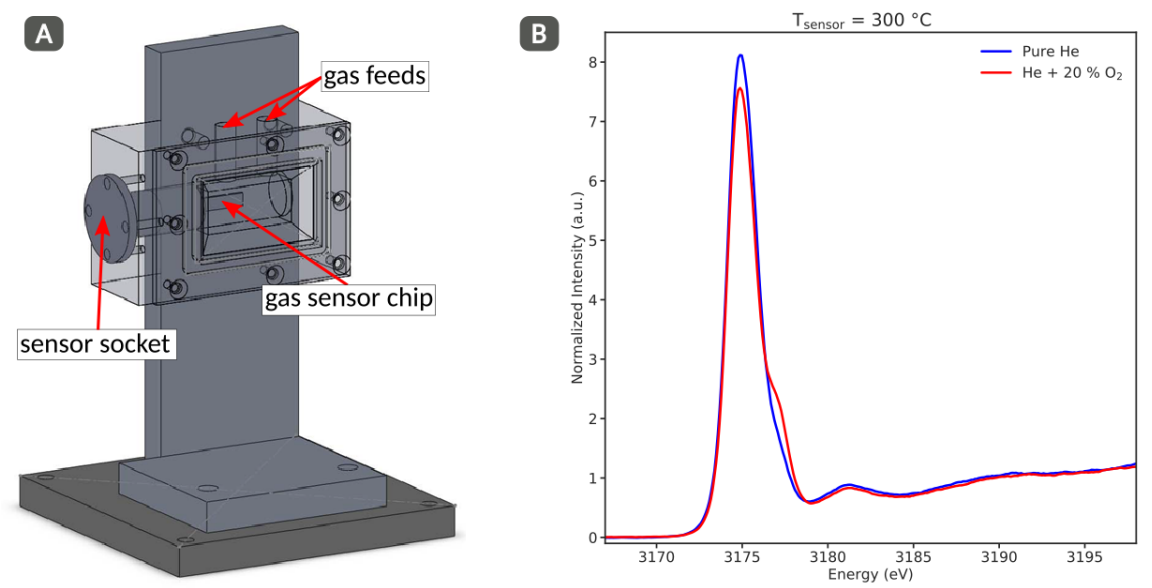

Figure S8. Schematic set-up of the operando XAS cell for gas sensors (A) and measurements at Pd $\mathrm{L}_{3}$-edge.

Journal of Instrumentation 9, C12017 (2014).

${ }^{S 6}$ T. Donath, S. Brandstetter, L. Cibik, S. Commichau, P. Hofer, M. Krumrey, B. Lüthi, S. Marggraf, P. Müller, M. Schneebeli, C. Schulze-Briese, and J. Wernecke, Journal of Physics: Conference Series 425, 062001 (2013).

S7I. Klačková, G. Blaj, P. Denes, A. Dragone, S. Göde, S. Hauf, F. Januschek, J. Joseph, and M. Kuster, Journal of Instrumentation 14, C01008 (2019).

${ }^{\mathrm{S} 8}$ W. M. Holden, O. R. Hoidn, G. T. Seidler, and A. D. DiChiara, Review of Scientific Instruments 89, 093111 (2018).

${ }^{\mathrm{S} 9}$ M. S. Haro, F. A. Bessia, M. Pérez, J. J. Blostein, D. F. Balmaceda, M. G. Berisso, and J. Lipovetzky, Radiation Physics and Chemistry, 108354 (2019).

${ }^{S} 10$ G. F. Knoll, Radiation Detection and Measurement, 3rd ed. (2000).

${ }^{511}$ N. BARSAN, D. KOZIEJ, and U. WEIMAR, Sensors and Actuators B: Chemical 121, 18 (2007).
${ }^{\mathrm{S} 12}$ D. Degler, Sensors 18, 3544 (2018).

${ }^{\mathrm{S} 13}$ D. Degler, S. Rank, S. Müller, H. W. Pereira de Carvalho, J.-D. Grunwaldt, U. Weimar, and N. Barsan, ACS Sensors 1, 1322 (2016).

${ }^{S 14}$ M. Hübner, D. Koziej, M. Bauer, N. Barsan, K. Kvashnina, M. D. Rossell, U. Weimar, and J.-D. Grunwaldt, Angewandte Chemie International Edition 50, 2841 (2011).

${ }^{\mathrm{S} 15}$ O. V. Safonova, T. Neisius, A. Ryzhikov, B. Chenevier, A. M. Gaskov, and M. Labeau, Chemical Communications, 5202 (2005).

${ }^{S} 16$ D. Koziej, M. Hübner, N. Barsan, U. Weimar, M. Sikora, and J.-D. Grunwaldt, Physical Chemistry Chemical Physics 11, 8620 (2009).

${ }^{\mathrm{S} 17}$ A. Staerz, I. Boehme, D. Degler, M. Bahri, D. Doronkin, A. Zimina, H. Brinkmann, S. Herrmann, B. Junker, O. Ersen, J.-D. Grunwaldt, U. Weimar, and N. Barsan, Nanomaterials 8, 892 (2018).

${ }^{S} 18$ N. Barsan and U. Weimar, Journal of Physics: Condensed Matter 15, R813 (2003).

${ }^{\mathrm{S} 19}$ M. Rovezzi, C. Lapras, A. Manceau, P. Glatzel, and R. Verbeni, Review of Scientific Instruments 88, 013108 (2017). 


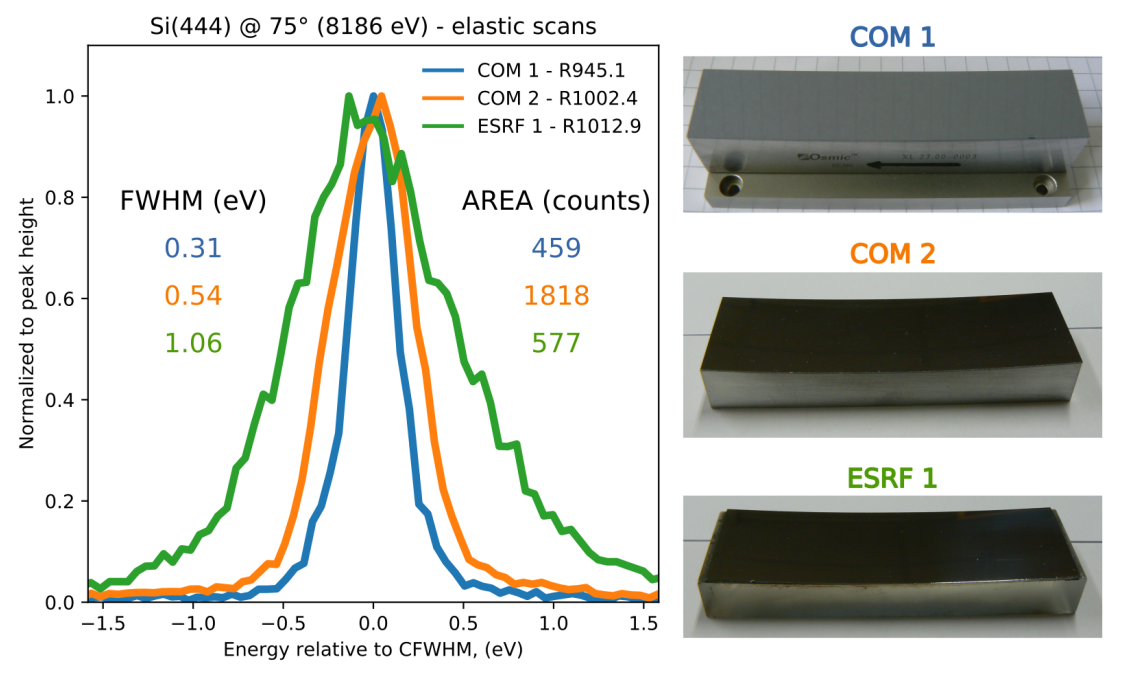

Figure S9. Elastic peak scans normalised to the peak height (left panel) for two commercially available Johansson analyzers ("COM 1" and "COM 2") versus one at the ESRF ("ESRF 1").

Single machining
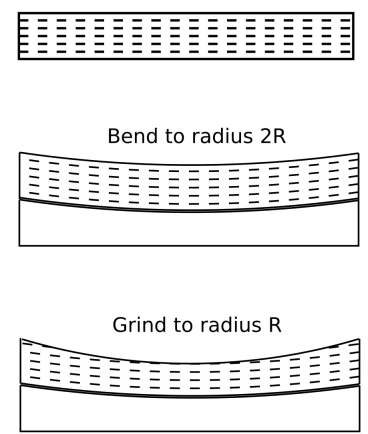

Double machining

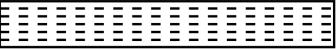

Grind to radius $2 \mathrm{R}$ (concave)

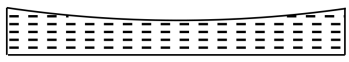

Grind to radius $2 \mathrm{R}$ (convex)

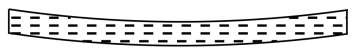

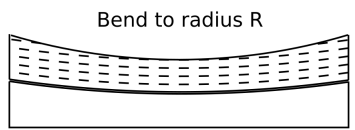

Figure S10. Machining approaches in Johansson crystal analyzers production: single machining versus double machining. The dashed lines represent the atomic planes. Dimensions are not in scale. 


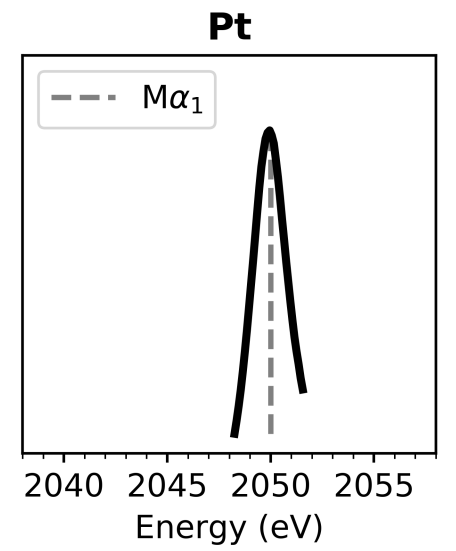

Hg

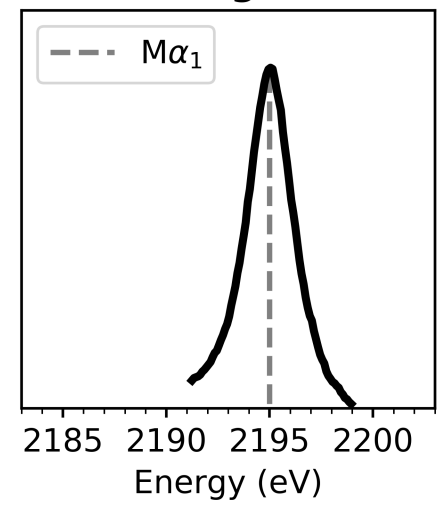

Rh

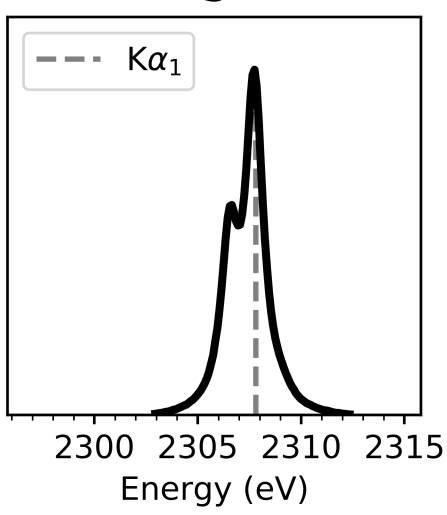

Ag

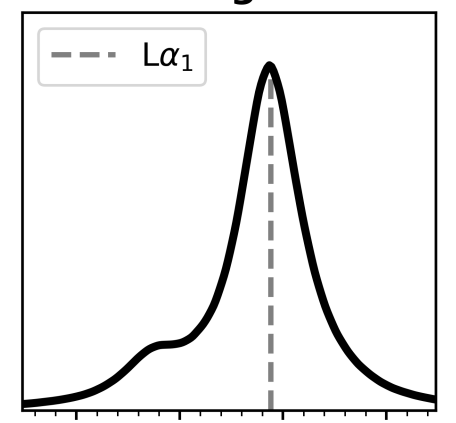

2975298029852990

Energy (eV)

Sn

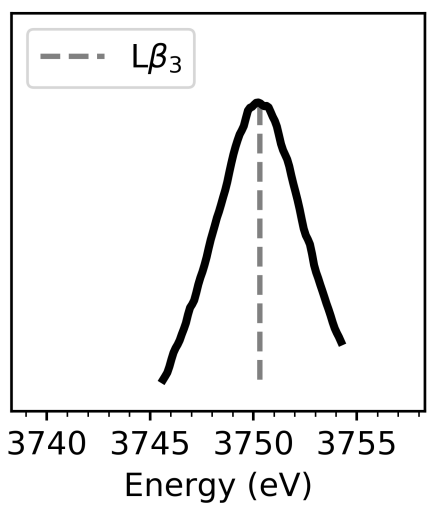

Sc

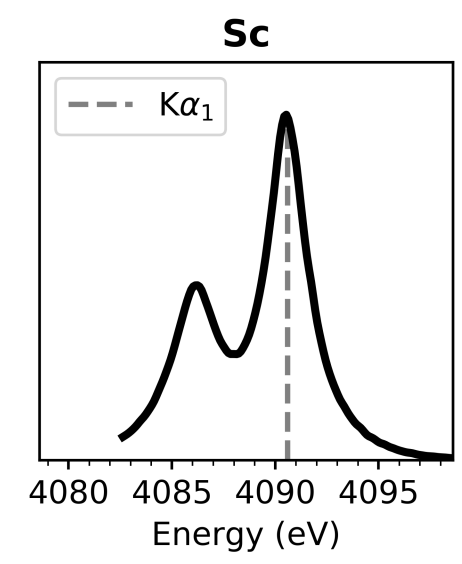

Cd

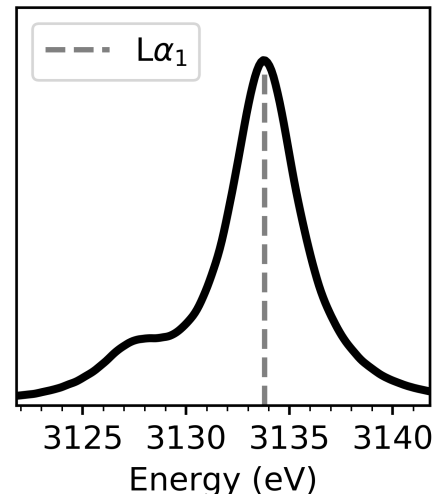

$y(e V)$
Mo

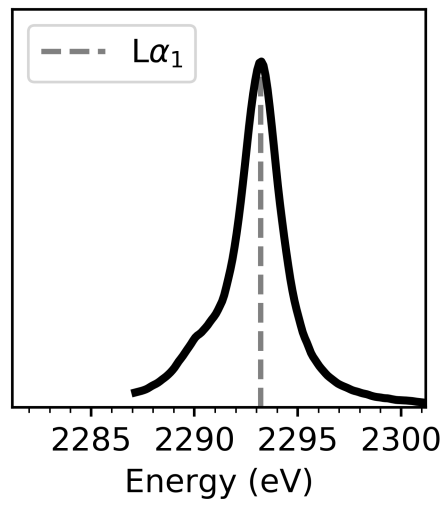

Pd

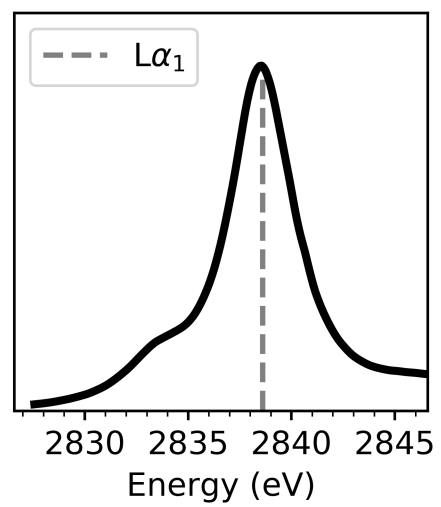

Sn

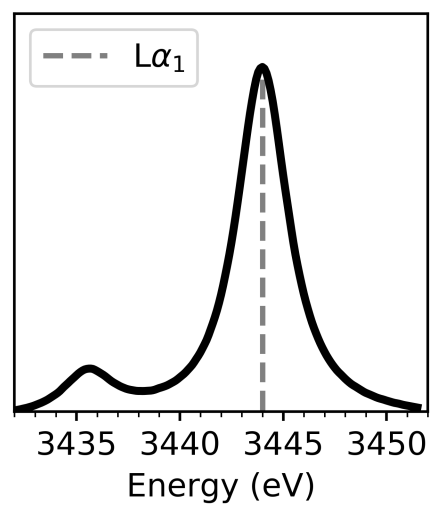

Figure S11. Measured emission lines. The energy range of all the plots is $20 \mathrm{eV}$. 
A

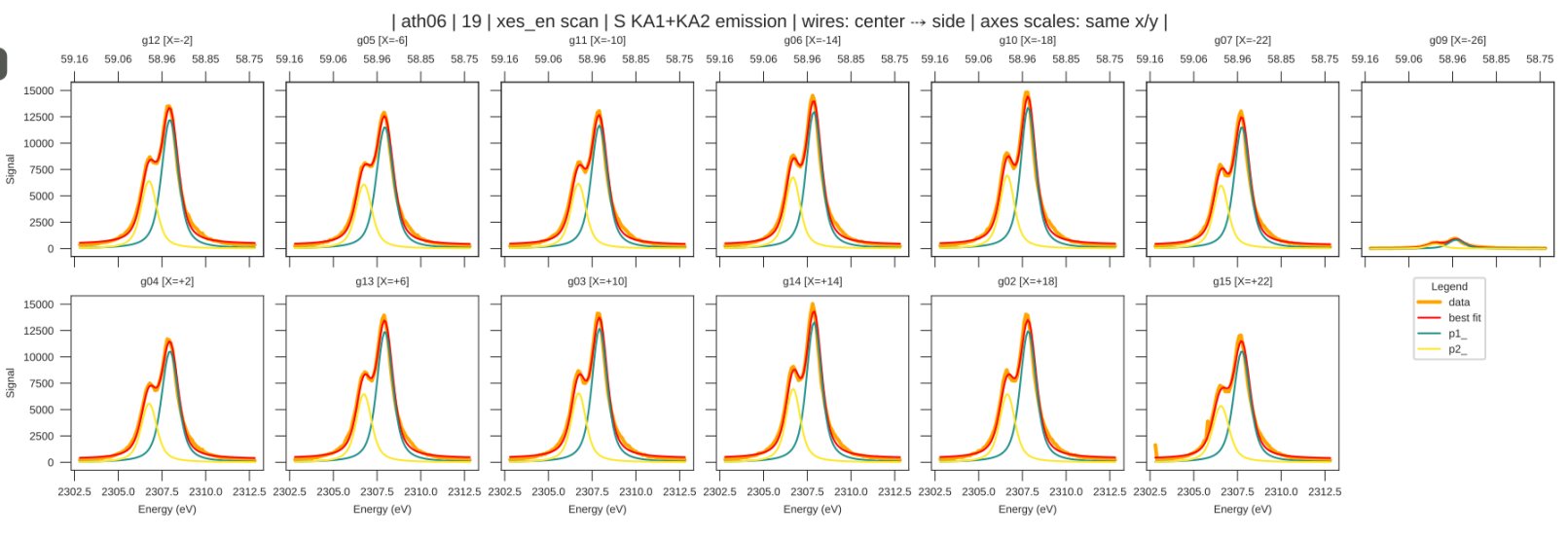

B
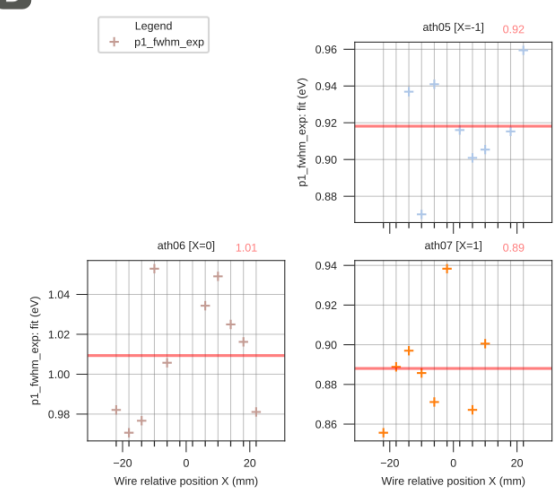

| xes_en | S KA1 emission | p1_fwhm_exp | aths: center $\rightarrow$ side | axes scales: same x(True)/y(False) |
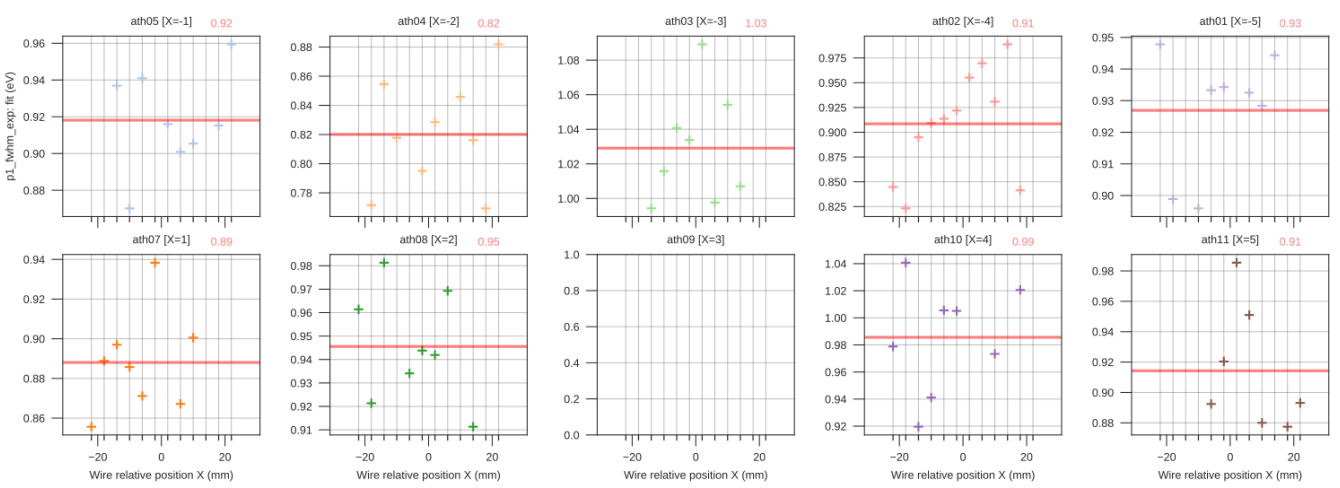

c

Summary of results for $\mathrm{S} K \alpha_{1}$

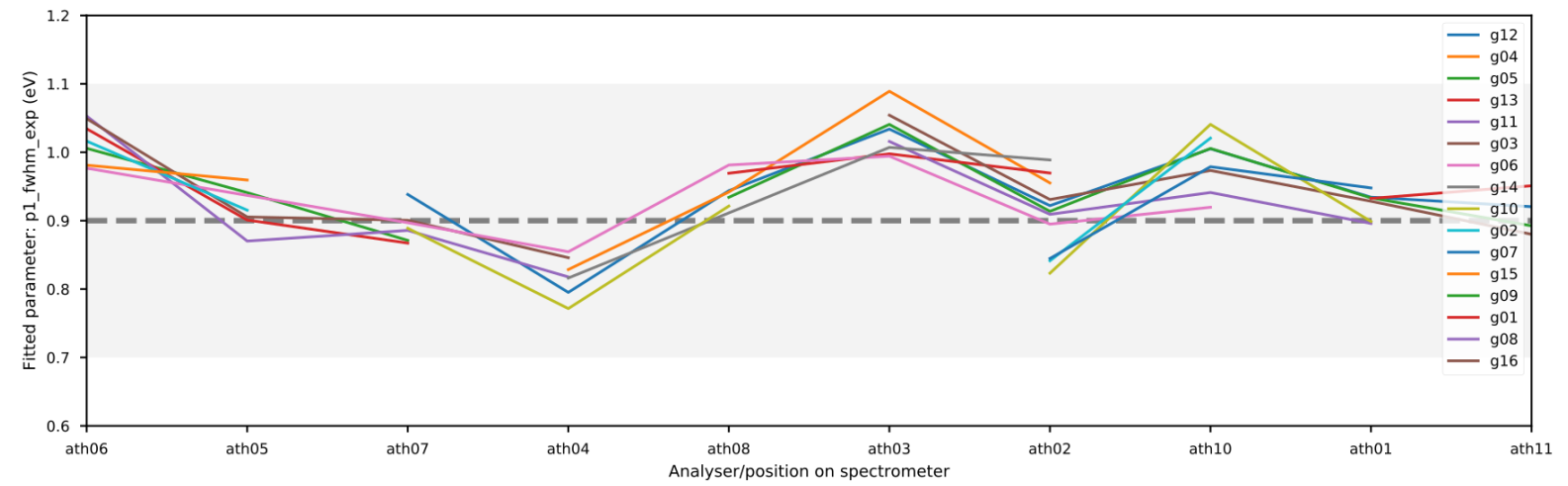

Figure S12. Data analysis example for S K $\alpha_{1,2}$ lines. Single-wire signal peak-fitting for a given analyzer, ath06 (A): the sub-plots have the same abscissa and coordinate scales. The distribution of the plots is from the central wires (g12, g04) to the side ones (g07, g15). The two rows of wires are symmetrically distributed. The signal from the side wires is partially cut by the detector entrance window. Evaluation of one fitted property, p1_fwhm_exp (B) and summary of the results (C). The horizontal dashed line is the average value and the gray area represents the experimental error bar. 
R parameter optmisation for each analyser

A) Raw data: collect emission scans at various $\mathrm{R}+$ peak fit individual wires signals.

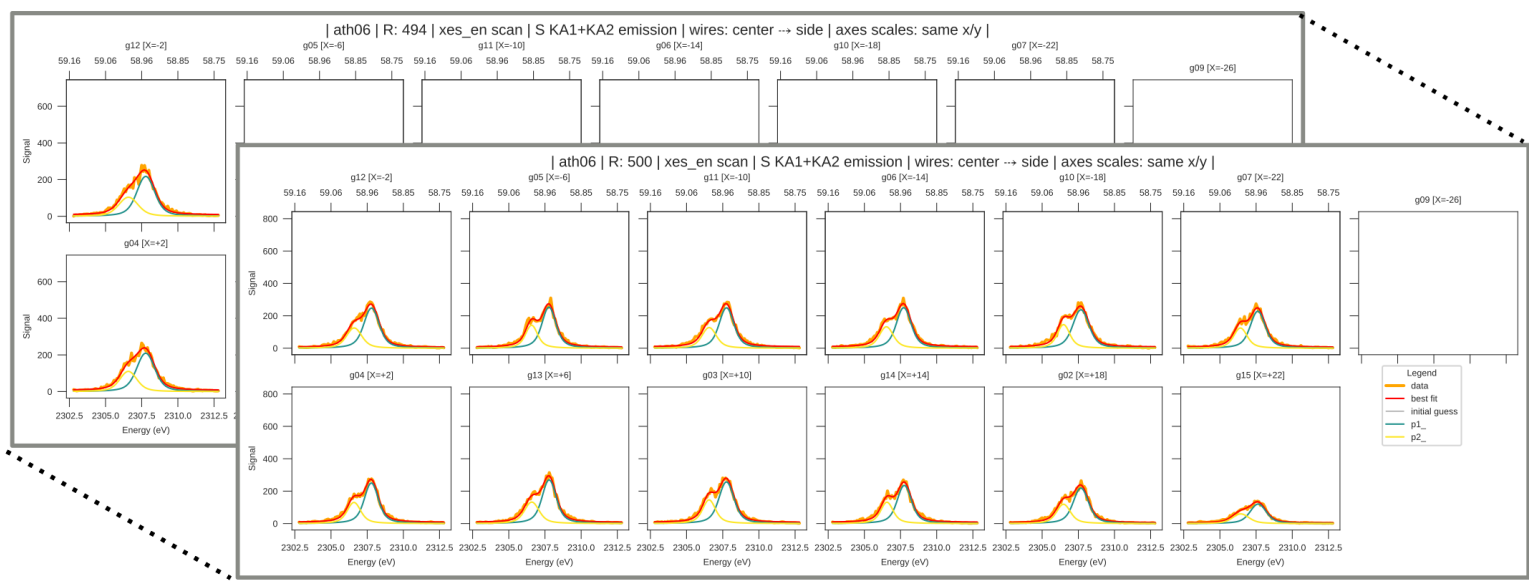

B) For each fitting parameter: perform quadratic fit to find best $\mathrm{R}$.

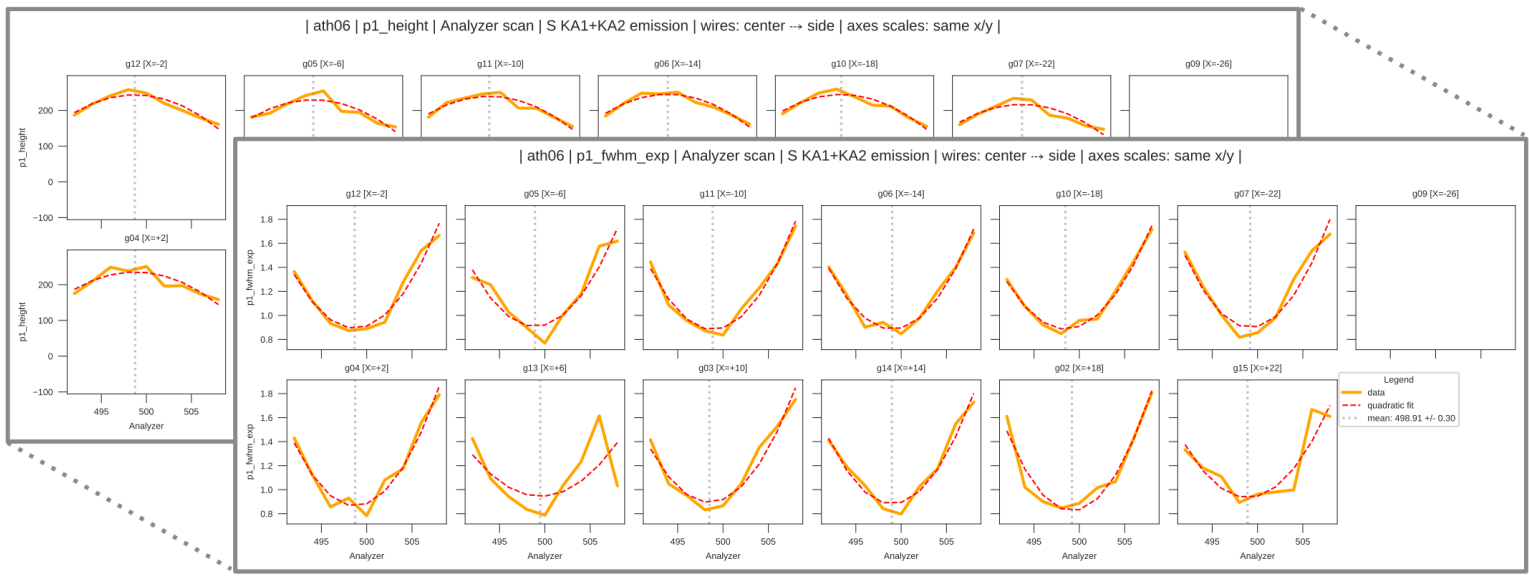

Figure S13. Procedure for finding the best R parameter for each crystal. Here the example of sulfur $\mathrm{K} \alpha_{1,2}$ lines at around $59^{\circ} \mathrm{Bragg}$ angle with $\mathrm{Si}(111)$ single-machined Johansson analyzers is shown. 